\title{
The Prediction and the Research on the Quality Risk of Supply Chain Based on Chaos Theory and PCAWOL Method
}

\author{
Liu Yang ${ }^{1}$ and Zhong $\mathrm{Wei}^{2}$ \\ ${ }^{1}$ Chongqing Technology \& Business Institute, Chongqing, China \\ ${ }^{2}$ Logistic Engineering University, Chongqing, China \\ Kris_ocean@163.com
}

\begin{abstract}
Along with the economic globalization, supply chain has broken the traditional regional limits. In this process, the risk factors which affect the quality of supply chain increases. The assessment for the quality risk of supply chain can prevent and control effectively the quality risk of supply chain. In this paper, we combine the principal component analysis and weight one-rank local-region method and propose a new prediction method-PCAWOL method on account of chaos theory. We use this method to predict the quality risk of the supply chain. The simulation shows that the method has a strong predictive ability and a more accurate prediction results for the quality risk of supply chain. It provides a new idea for the quality risk of supply chain.
\end{abstract}

Keyword: the quality risk of supply chain; PCAWOL; chaos theory

\section{Introduce}

With the arrival of the economic globalization and the intensification of the competition, a single enterprise is difficult to survive and develop in the fierce market competition. The market competition will be developed from the competition of the enterprises to the completion of supply chain [1-2]. A high level product quality and excellent service quality can create the satisfaction, enthusiasm and loyalty of the customers. The uncertain risk that the supply chain exists restricts the increase of supply chain's overall strength; especially the quality risk has become the key factors which effect the effective operation of supply chain. Therefore, according to studying the quality risk of supply chain, it has great significance to predict the quality risk of supply chain.

In foreign countries, many scholars have studied the quality risk of supply chain. According to the investigation and study, Hendricks thought the fluctuation of supply chain made the average wealth of the enterprises decreased 10.28\% [3]. Singhal found that the sudden risk of supply chain would lead to the income fell by an average $33 \%$ to $40 \%$ with a large number of enterprises [4]. Chu-Hua Kuel etc. thought that the information, relationship and innovation were the key factors to ensure the product quality and reduce the quality risk of supply chain [5]. In 2000, Stanle and other scholars defined the quality risk of supply chain as a new dimension in supply chain coordination. Game theory was also used to the relative questions about the quality risk of supply chain research and the evaluation and the selection [6]. Then, Stanley etc. discussed the two different contract agreements which were the acceptable quality level and the protocol efficiency under the group security between the manufacturers and the suppliers. Thus they brought the quality risk information into the research and coordination of supply chain [7]. In 2006, Christopher S.Tang studied the risk management of supply chain from four different angles. The four different angles are product, supply, demand and information [8]. In China, Wang Bangjun studied the quality risk of supply chain and its application. He introduced the practice of the quality risk management of supply chain and discussed the significance of 
the quality risk management of supply chain for the enterprises [9]. Jiang Jiadongetc studied the characteristics of the quality risk of supply chain. They divided the quality risk of supply chain into three forms and discussed the main features of the quality risk of supply chain. Finally, they prospected the development direction for the quality risk of supply chain [10]. Zhuang Hongxing studied the quality risk control for the industry link. The author took the dairy quality risk as the studied object and put forward the system view aiming to how to control the quality risk of industry link of the diary product. The author proposed to establish the quality traceability system. He used the risk assessment as the basis of the quality risk management and warning. And he used the quality traceability system to trace and recall the quality security [11]. Zhang Huaying used the aquatic product supply chain quality risk as the research object and discussed these from the quality risk identification, the quality risk evaluation and the quality risk controlthree aspects. He put forward the aquatic products quality risk management scheme which integrated a variety of economic management tools [12].

Chaos science is an important branch and research point in the nonlinear science. It is a singular steady state behavior in the nonlinear dynamic system. It characterizes a kind of essential feature characterization of the complex phenomenon which exists in nature and human society. Chaotic prediction is one of the important research directions in the chaos. Its application characteristics use the characteristics of the chaotic motion. And it overcomes the defect of the traditional prediction and makes the prediction results more superior. Chaotic prediction has been applied in many fields. And it has achieved remarkable economic and social benefits. The advocated person of chaos science M.Shlesingle and the famous physicist J.ford thought that chaos is the third revolution about the twentieth century physics [13]. In 1971, Ruelle and Takens found a strange attraction in the nature of turbulence. And they proved that the motion which had the strange attractor was the chaotic motion [14]. In 1975, Li Tianyan and J.Yorke published the period three implies chaos, gave the Li-Yorke theory and introduced the chaos [15]. In 20 twentieth centuries 90, Ott E, Pecora and Carroll have made breakthrough progress in chaos control and chaos synchronization [16-17]. Nearly twenty years, while the chaos theory promoted other disciplines development, in turn, the development of other disciplines promoted the depth and extensive research on the chaotic theory. This promoted greatly counterchange, permeation and promotion between the chaos and other disciplines [18]. HarhadKhellat, AkasheGhaden and NastaranVasegh investigated a globally non-local coupled map lattice. They proved the existence of chaos in the scene of $\mathrm{Li}$-Yorke in that system is presented in terms of the Marotto theorem. Then, they analyzed this system was chaotic and determined the synchronous behaviors. [19]. Aline Souza de Paula and Marcelo AmorimSavi presented an overview of chaos control methods classified as follows: OGY methods also included discrete and semi-continuous approaches; and time-delayed feedback methods that were continuous approaches. These methods were employed in order to stabilize some desired UPOs establishing a comparative analysis of all methods [20].

In this paper, firstly, we introduce the research statue of the quality risk of supply chain and chaos prediction theory. Secondly, we define the quality risk of supply chain and the quality risk classification for supply chain. Thirdly, we combine the principal component analysis and weight one-rank local-region method to propose a new chaotic prediction method-PCAWOL method. Finally, we carried out the numerical experiments and predicted the dynamic risk for the quality risk of supply chain. The construction of this paper is as follows. The first part is the introduction. The second part is the quality risk analysis of supply chain. In this part, we analyze the quality risk of supply chain and class the level for quality risk analysis of supply chain. The third part is the improved chaotic prediction method. In this part, we present an improved chaotic prediction method-PCAWOL method. The fourth part is the number analysis and the last part is the conclusion. 


\section{The Quality Risk Analysis of Supply Chain}

\subsection{The Quality Risk Identification of Supply Chain}

2.1.1 The Definition for the Quality Risk of Supply Chain: Although the quality risk question of supply chain has paid more and more attention, there were plenty of literatures studying the quality risk question of supply chain. However, there was not a clear definition for the quality risk of supply chain. It led to the diversity, surface and non-systematic for the quality risk of supply chain. Generally speaking, the quality risk refers to the possibility that the negative quality events happened or not happened in future. Among them, the negative quality events refer to the events that the quality managers do not want to happy, will lead to the quality loss and bring other bad results. The quality risk exists in all of the quality management activities. And it leads to the uncertainty that the negative event happens. The quality risk is determined by two characteristics. The two characteristics belong to the negative quality event that may happen in future. For the two characteristics, one is the probability that the negative quality event happens. The other is the result that the negative quality event brings. Due to the diversity of the negative quality event speculation, the quality risk assessment is different from other risk evaluation. Under the environment of supply chain, the quality risk of supply chain is the sum of all kinds of quality risk which faces in the related links.

2.1.2 The Characteristics for the Quality Risk of Supply Chain: Various of complex uncertain factors lead to various kinds of uncertain risks in supply chain, such as natural risk, supply and demand risk, moral risk, system risk, information transfer risk and price risk. The quality risk is the basic risk in supply chain risk. Many risks in supply chain risk show that the product quality is fluctuation abnormally. This fluctuation leads to the customer satisfaction decreased and makes the enterprise damaged. Compared with other risks for supply chain, the quality risk of supply chain has the following characteristics.

The first is the transmission of the quality risk. The quality risk of supply chain can transfer from the front raw material suppliers to the last consumers along the transmission direction of supply chain logistics (that is also product flow). The transfer process is not a simple linear process. In transfer process, the quality risk may bring about many changes and expose the unexpected quality question. In addition, due to the complexity of supply chain risk, the link that appears the quality question is not the source for the quality risk of supply chain sometimes.

The second is the outbreak delay of the quality risk. In supply chain, many risks, such as supply and demand risk and price risk, often show quickly with the change of the market competition situation. The quality risk has some aura identification features. However, it has a period of latency. When come environmental conditions have, the quality risk may outbreak. Once outbreak, it shows that a variety of non-expected quality risk incidents of supply chain.

The third is the relevance of the quality risk factors. The quality risk of supply chain does not exist in isolation. It has an interaction with other risks. The price risk and the quality risk of supply chain have a close relationship. The quality risk of supply chain and other risks are interaction. Therefore, other risks in supply chain can convert to the quality risk. And the quality risk can also convert to the cost in manufacturing enterprise and time risk under certain conditions.

The fourth is the controllable of the quality risk. The quality risk of supply chain often occurs within supply chain and most of them are artificial. In general, the occurrence of the quality risk needs the accumulation of factors and certain occurrence conditions. We need to identify and control the formation and accumulation of the risk factors. In addition, we also need to avoid the necessary conditions that can lead to the occurrence of the quality risk. Then we can control effectively the occurrence for the quality risk event of supply 
chain. By changing the condition that can lead to the occurrence of the quality risk, we can transform of resolve the quality risk of supply chain. On the contrary, the quality risk will produce bullwhip effect according to transmission.

The fifth is the serious consequences of the quality risk. When the consumer select and purchase products, they consider the quality firstly. Once the occurrence of the quality risk of supply chain, it will influence the market share of the market and the market reputation of the related market. Seriously, it will lead to the related enterprises bankruptcy and even the fracture of supply chain. Therefore, the consequence of the quality risk of supply chain has the characteristics of wide influence and great loss.

\subsection{The Quality Risk Classification for Supply Chain}

In this paper, we divide the quality risk classification for supply chain into 5 second index. They are the collaboration risk of supply chain, the structure risk of supply chain, the operation risk of supply chain, the quality risk of product and external factors. Every second index was still classed into the third index.

Tabel 1. The Quality Risk Classification for Supply Chain

\begin{tabular}{|c|c|c|}
\hline The first index & The second index & The third index \\
\hline \multirow{21}{*}{$\begin{array}{c}\text { The quality } \\
\text { risk of supply } \\
\text { chain }\end{array}$} & \multirow{5}{*}{$\begin{array}{c}\text { The collaboration risk of } \\
\text { supply chain }\end{array}$} & Information share \\
\hline & & contract \\
\hline & & Partners trust \\
\hline & & Supplier selection criteria \\
\hline & & The relationship with suppliers \\
\hline & \multirow{5}{*}{$\begin{array}{c}\text { The structure risk of } \\
\text { supply chain }\end{array}$} & The length of supply chain \\
\hline & & The number of supply chain \\
\hline & & The structure of supply chain node enterprise \\
\hline & & The structure of supply chain \\
\hline & & The structure of enterprise staff \\
\hline & \multirow{5}{*}{$\begin{array}{c}\text { The operation risk of } \\
\text { supply chain }\end{array}$} & The operation of supply chain \\
\hline & & The operation of supplier \\
\hline & & The operation of manufacturer \\
\hline & & The operation of retailer \\
\hline & & The operation of logistics center \\
\hline & \multirow{4}{*}{$\begin{array}{l}\text { The quality risk of } \\
\text { product }\end{array}$} & The product innovation \\
\hline & & The product security \\
\hline & & The product appearance \\
\hline & & The product manufacture \\
\hline & \multirow{2}{*}{ External factors } & Government policy \\
\hline & & Natural factors \\
\hline
\end{tabular}

According to the quantitative method, we refined the quality risk of supply chain. We adopted 10 points to refine. The bigger the score value is, the higher the risk degree is. The specific grading standard is as follows.

Tabel 2. The Specific Grading Standard

\begin{tabular}{|c|c|c|}
\hline $\begin{array}{c}\text { The risk } \\
\text { grade }\end{array}$ & Score system & \begin{tabular}{c} 
Influence on the quality of supply chain \\
\hline Level 1
\end{tabular} 0,1,2 \\
\hline Level 2 & $2,3,4$ & The risk can be ignored \\
\hline Level 3 & $4,5,6$ & $\begin{array}{c}\text { Slight risk and low level } \\
\text { supply chain and needs to take measure to prevent the }\end{array}$ \\
\hline
\end{tabular}




\begin{tabular}{|c|c|c|}
\hline & & risks. \\
\hline Level 4 & $6,7,8$ & $\begin{array}{c}\text { Very high level risk, the serious consequence and causes } \\
\text { the serious damage to the quality of supply chain }\end{array}$ \\
\hline Level 5 & $8,9,10$ & $\begin{array}{c}\text { The highest level risk and causes the devastating damage } \\
\text { to the quality of supply chain }\end{array}$ \\
\hline
\end{tabular}

\section{The Improved Chaos Prediction Method}

\subsection{The Chaotic Identification}

Before the application of the chaotic theory, we need to determine that whether the original time series has the chaotic characteristics or not. Then, we can use the chaotic theory. Chaos is a random behavior which does not to need any additional random factors in the definite nonlinear dynamic system. There are several methods which are used to determine whether a system has the chaos or not. They are Lyapunov index, Kolmogorov entropy, topological entropy, the correlation dimension and the power spectrum etc. the largest Lyapunov index is the widely used method which is based on the Wolf method.

We assume that the chaotic time series is $x(1), x(2), \cdots x(t)$, and we reconstruct the phase space.

$X(t)=\{x(t), x(t+\tau), \cdots x[t+(m-1) \tau]\}, t=1,2, \cdots, M$

We assume that the initial point is $X\left(t_{0}\right)$. The distance is $L_{0}$ which is nearest to the neighbor point $X_{1}\left(t_{0}\right)$. We track the time evolution of the two points until $t_{i}$ time. If the spacing exceeds the predetermined threshold value $\varepsilon(\varepsilon>0)$ and $L_{0}^{\prime}=\left|X\left(t_{1}\right)-X\left(t_{0}\right)\right|>\varepsilon$, we retain $X\left(t_{1}\right)$. Then, we find another point $X_{1}\left(t_{1}\right)$ in $X\left(t_{1}\right)$ neighborhood which is made $L_{1}=\left|X\left(t_{1}\right)-X_{1}\left(t_{1}\right)\right|<\varepsilon$ and the included angle is as small as possible. We repeat the above process until the $x(t)$ arrives the end $N$ of the time series. The total iteration number of the tracing and evolutionary process is $t_{M}-t_{0}$. Therefore, the largest Lyapunovindex $\lambda_{1}$ is:

$\lambda_{1}=\frac{1}{t_{M}-t_{0}} \sum_{t=0}^{M} \operatorname{In} \frac{L_{i}^{\prime}}{L_{i}}$

Among it,

$L_{i}^{\prime}=\left|X\left(t_{i}\right)-X\left(t_{i-1}\right)\right|, L_{i}=\left|X\left(t_{i}\right)-X_{i}\left(t_{i}\right)\right|$

Under the state $X\left(t_{i}\right), X_{i}\left(t_{i}\right)$ is one point in $t_{i}$ time that the radius is $\varepsilon$.

\subsection{The Principal Component Analysis}

The principal component analysis method is to introduce firstly the non-random varialbes by Kart Pearson. Then, it is extended to the random variables by Hotelling. The principal component analysis method uses the method which reduces the dimension in the statistical analysis. This method applied to the analysis of multi index. In the economic field, in order to analyze the problems comprehensively, we need to put forward many indexes which can reflect the problems in different degrees. When we use the multivariate statistical to analyze the multi-index problem, it will increase the complexity because the indexes are too many. Therefore, we hope that the number of the indexes is small and we can get more information. In many cases, the criteria are related. The information that the criteria reflect has a degree overlap. The principal component analysis is to establish some new indexes as little as possible for the original indexes. It makes that the new indexes are not related. And the new indexes reflect the problems maintain the original information as much as possible 
The principal component analysis reconstructs the original related indexes to the new nonrelated indexes. In general, the mathematical modeling is to do the linear combination for the original $p$ indexes which are as the new comprehensive indexes. The classical approach is to use the largest $\operatorname{Var}\left(F_{1}\right)$ of first linear combination $F_{1}$ to express. It expresses the information of $F_{1}$ is much. Therefore, in all linear combinations, the variance of $F_{1}$ is the biggest. $F_{1}$ is the first principal component. If the first principal component cannot express the information of the original $p$ indexes, we select the second linear combination $F_{2}$. In order to reflect the original information, the information of $F_{1}$ cannot appear in $F_{2}$. That is, $\operatorname{Cov}\left(F_{1}, F_{2}\right)=0$. We call $F_{2}$ as the second principal component. We repeat the above process and get $p$ principal components.

\subsection{The Weighted One-rank Local-region Method}

The weighted one- rank local-region method makes the last point in the reconstruction phase space as the central point. And it makes the trajectory point which is nearest from the central point as the related point. According to the evolution law of the trajectory, we make the forecast. This method has better prediction ability and higher prediction accuracy.

The first is to make the time series $x(t), t=1,2, \cdots, N$.

The second is to recon structure the phase space according to calculate the time delay and the embedding dimension.

$Y(t)=(x(t), x(t+\tau), \cdots, x(t+(m-1) \tau)) \in R^{m}, t=1,2, \cdots, M$

Among them, $M$ is the number of the reconstruction phase space.

$$
M=N-(m-1) \tau
$$

The third is to find the near point. We calculate the distance between each point in space and the center point $Y_{k}$. And we need to find the reference vector set $Y_{k i}, i=1,2, \cdots, q$ for $Y_{k}$. We define the distance between $Y_{k i}$ and $Y_{k}$ as $d_{i}$. We resume $d_{m}$ is the minimum value in $d_{i}$ and define the weight value of $Y_{k i}$ as

$P_{i}=\frac{\exp \left(-a\left(d_{i}-d_{m}\right)\right)}{\sum_{i=1}^{q} \exp \left(-a\left(d_{i}-d_{m}\right)\right)}$.

$a$ is parameter and $a=1$.

The fourth is to forecast. The weighted one-rank local-region method can fit it as

$$
\left[\begin{array}{l}
Y_{k 1+1} \\
Y_{k 2+1} \\
\cdots \\
Y_{k q+1}
\end{array}\right]=\left[\begin{array}{l}
e Y_{k 1} \\
e Y_{k 2} \\
\cdots \\
e Y_{k q}
\end{array}\right]\left[\begin{array}{l}
a \\
b
\end{array}\right] .
$$

Among them, $e=(1,1, \cdots, 1)^{T}$ and $a, b$ is coefficient.

When $m \geq 1$,

$$
\left[\begin{array}{l}
x_{k 1+1} \\
x_{k 2+1} \\
\cdots \\
x_{k q+1}
\end{array}\right]=\left[\begin{array}{l}
e x_{k 1} \\
e x_{k 2} \\
\cdots \\
e x_{k q}
\end{array}\right]\left[\begin{array}{l}
a \\
b
\end{array}\right] .
$$

According to the least squares method, we get 
$\sum_{i=1}^{q} P_{i}\left(x_{k i+1}-a-b x_{k i}\right)^{2}=\min$

The above function is two variables for $a$ an $b$. Derivativeit, we get

$\left\{\begin{array}{l}\sum_{i=1}^{q} P_{i}\left(x_{k i+1}-a-b x_{k i}\right)=0 \\ \sum_{i=1}^{q} P_{i}\left(x_{k i+1}-a-b x_{k i}\right) x_{k i}=0\end{array}\right.$

For short, we get

$\left\{\begin{array}{l}\left.a \sum_{i=1}^{q} P_{i} x_{k i+1}+b \sum_{i=1}^{q} P x_{k i}\right)^{2}=\sum_{i=1}^{q} P x_{k i} x_{k i+1} \\ a+b \sum_{i=1}^{q} P x_{k i}=\sum_{i=1}^{q} P x_{k i+1}\end{array}\right.$

According to the function, we obtain $a$ an $b$. And we take $a$ an $b$ into (8).

The fifth is to forecast according to the prediction function. Among them, reference vector $Y_{k i}, i=1,2, \cdots, q$ is the first prediction. $Y_{k i+1}, i=1,2, \cdots, q$

The sixth is to define the error and establish the result $x(t)$.

$E r=\frac{\sqrt{\sum_{i=1}^{k}(x(t)-x(t))^{2}}}{\sqrt{\sum_{i=1}^{k} x^{2}(t)}} \cdot 100 \%$

\section{Numerical Analysis}

We use PCAWOL method to predict the quality risk of supply chain for one enterprise. We take the first 1000 time series data that we collect as the training set and take the last 200 time series data as the sample set. Firstly, we use the principal component analysis method to calculate the expression of the principal component analysis that the time series of supply chain quality risk corresponds. We divide the quality risk of supply chain into five second indexes and twenty-first third indexes. Supply chain collaboration risk, supply chain structure risk, supply chain operation risk, product quality risk and the exterior factors are recorded as $\left\{X_{1}\right\},\left\{X_{2}\right\},\left\{X_{3}\right\},\left\{X_{4}\right\}$ and $\left\{X_{5}\right\}$. The time series for the quality risk of supply chain is recorded as $\left\{X_{i}\right\}$. The result of the principal component analysis is shown in the following table.

Table 3. The Result of the Principal Component Analysis

\begin{tabular}{|c|c|c|c|}
\hline $\begin{array}{c}\text { Principal } \\
\text { Component }\end{array}$ & $\begin{array}{c}\text { Characteristic } \\
\text { Root }\end{array}$ & $\begin{array}{c}\text { Contribution } \\
\text { Rate }(\%)\end{array}$ & $\begin{array}{c}\text { Cumulative } \\
\text { Contribution Root }(\%)\end{array}$ \\
\hline 1 & 6.36 & 28.43 & 29.43 \\
\hline 2 & 3.95 & 17.65 & 46.08 \\
\hline 3 & 4.05 & 18.11 & 64.19 \\
\hline 4 & 7.33 & 32.78 & 96.97 \\
\hline 5 & 0.68 & 3.03 & 100 \\
\hline
\end{tabular}


According to the principal component analysis, we can get the expression of the principal component analysis that the time series of supply chain quality risk.

$$
X_{i}=0.28 X_{1}+17.6 X_{2}+18.1 X_{3}+0.33 X_{4}
$$

Then, we need to obtain the Lypaunov indexes of $\left\{X_{1}\right\},\left\{X_{2}\right\},\left\{X_{3}\right\},\left\{X_{4}\right\},\left\{X_{5}\right\}$ and $\left\{X_{i}\right\}$. And we get the largest Lyapunov index. These indexes are $\left\{X_{1}\right\}=0.0043$, $\left\{X_{2}\right\}=0.0038,\left\{X_{3}\right\}=0.0009,\left\{X_{4}\right\}=0.0033$ and $\left\{X_{i}\right\}=0.0076$. We can see that the largest Lyapunov indexes are more than zero. Therefore, the quality risk time series of supply chain has the chaotic characteristics and we can use the chaotic prediction method to predict.

Then, we use c-c method to get the embedded dimension and delay time. The embedded dimension $m=5$ and delay time $\tau=7$.

After we get the time delay and the embedding dimension, we use the PACBG method to predict the quality risk of supply chain. The results are as follows.

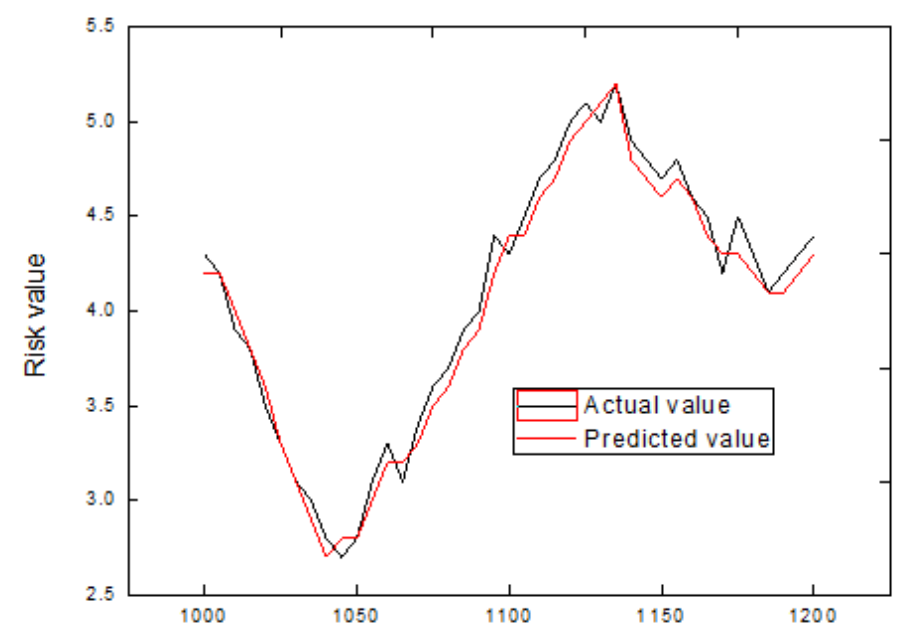

Figure 1. The Results of the Prediction Value and the Actual Value

From the above figure, we can see that the PCAWOL method has good prediction effect. Its prediction is more accurate for the quality risk of supply chain and has little error. The predicted result is more ideal.

\section{Conclusion}

The loss that the quality risk of supply chain causes becomes greater for the supply chain. According to evaluate and predict the quality risk of supply chain, it can reduce effectively the probability of the quality risk of supply chain. Then it can reduce the loss for supply chain. In this paper, we do a bit of work. Firstly, we identify respectively the quality risk of supply chain. Secondly, we establish the assessment system for the quality risk of supply chain. Thirdly, in order to predict the quality risk of supply chain better, we put forward the PCAWOLmethod which is based on the chaotic prediction theory. Finial, we apply the PCAWOL method to the prediction for the quality risk of supply chain. The experimental results show that the prediction accuracy is more precise and the error is less. The predicted result is more ideal. 


\section{References}

[1] K. K. Boyer, "Extending the Supply Chain", [M]. New York: AMACOM, (2005).

[2] D. J. Ketchen and L. C. Giunipero, "The Intersection of Strategic Management and Supply Chain Management", [J] . Industrial Marketing Management, vol. 33, no. 1, (2004), pp. 51-56.

[3] K. B. Hendricks and V. R. Singha, "An empirical analysis of the effect of supply chain disruptions on long-run stock price performance and equity risk of the firm", $[\mathrm{J}]$. Production and Operations Management, vol. 14, no. 1, (2005), pp. 5-52.

[4] V. R. Singhal, "The effect of supply chain glitches on shareholder wealth", [J]. Journal of Operation Management, vol. 21, no. 5, (2003), pp. 501-522.

[5] C. H. Kuel and C. N. Madu, "How to outperform Competitors with SCQM", [D]. The Lutin School of Business of Pace University, (2003).

[6] B. Stanley, P. E. Fishcher and M. V. Rajan, "Information, Contracting and Quality Cost", [J]. Management Science, vol. 46, no. 6, (2000), pp. 776-789.

[7] S. Bainman and S. Netessine, "Howard Kunreuther Procerement in supply Chains when the End-Product Exhibits the "Weakest Link" Property", [D], The Wharton School, University of Pennsylvania, (2004).

[8] C. S. Tang, "Perspectives in supply chain risk management", International Journal of Production Economics, no. 10, (2006), pp. 451-488

[9] W. Bangjun, "The risk management of the supply chain quality and the application", [J]. Aeronautic Standardization \& Quality, vol. 1, (2005), pp. 12-16.

[10] J. Jiadong, Z. Hanping and F. Yuncheng, "Study on the features of quality risk in supply chain", [J]. Aeronautic Standardization \& Quality, vol. 223, (2007), pp. 29-34.

[11] Z. Hongxing, "Research on diary industrial chain quality risk control based on quality traceability system", [D]. Shandong University, management science and engineering, (2012).

[12] Z. Huaying, "Research on quality risk management of aquatic supply chain under farming-supermarket docking", [D]. South China University of Technology, Logistics Engineering and Management, (2013).

[13] C. Shihua and L. Junan, "Chaotic dynamics", Wuhan, Wuhan University of hydraulic and electric power press, (1998).

[14] D. Ruelle and F. Takens, "On the natural of turbulence”, [J].Commum Math Phys., vol. 20, no. 1, (1971), pp. 167-192.

[15] T. Y. Li and J. A. Yorke, "Period three impliechaos", [J], Amer. Math. Monthly, vol. 82, no. 10, (1975), pp. 985-992.

[16] E. Ott, C. Grebogi and J. A. Yorke, "Controlling Chaos", [J]. Phys. Rev. Lett, (1990), vol. 64, no. 11, pp. 1196-1199.

[17] L. M. Pecora and T. L. Carroll, "Synchronization in chaotic systems", [J]. Phys. Rev. Lett. vol. 64, no. 8, (1990), pp. 821-825.

[18] H. Peisheng, H. Rensheng and H. Hao, "Chaos analysis and its application", [M]. Wuhan: Wuhan University Press, (2005).

[19] H. Khellat, A. Ghaden and N. Vasegh, "Li-Yorke chaos and synchronous chaos in a globally nonlocal coupled map lattice", [J]. Chaos, Solitons \& Fractals, vol. 44, no. 11, (2011), pp. 934-939.

[20] A. Souza de Paula and M.AmorimSavi, "Comparative analysis of chaos control methods: A mechanical system case study", [J]. International Journal of Non-Linear Mechanics, vol. 46, no. 8, (2011), pp. $1076-1089$.

[21] Z. Xueqing and L. Jun, "Chaotic characteristics analysis and prediction model study on wind power time series", [J]. Acta Phys. Sin, vol. 61, no. 5, (2012), pp. 7-12.

[22] L. Danayng, W. Yawei, W. Xian, H. Kun, Z. Xingjuan and Y. Chunxin, "Chaotic property analysis of pressure fluctuation for oxygen phase change heat exchanger", [J]. Acta Phys, (2012), vol. 61, no. 15, pp. $1-7$

[23] L. Jinhu, L. Jun-an and C. Sshihua, "The Analysis and Applications of Chaotic Time Series", [M], Wuhan: Wuhan University Press, (2005)

[24] M. Juan and X. Peikai, "Load Forecasting Based on An Improved Weighted First-Order Model", [J]. Techniques of Automation and Application, vol. 28, no. 9, (2009), pp. 15 -17.

[25] Z. Jun, W. Huan and C. Zhaofa, "The improved weighted first-order local multi-step prediction model", [J]. Statistics and Decision, vol. 16, no. 33, (2008).

[26] Y. Liming, "An improved immune genetic algorithm and the application in optimal design of PID controllers", [D]. Central South University, control theory and control engineering, (2007). 
International Journal of $u-$ and e- Service, Science and Technology Vol.9, No. 6 (2016) 